\title{
Research on Line Loss Calculation Methods Based on DC Power Flow Models
}

\author{
FAN Li ${ }^{1, a}$, FAN Zhen ${ }^{2, b}$, WU Yaowu ${ }^{2, c}$, LOU Suhua ${ }^{2, d}$, WEI Zhen ${ }^{2, ~ e ~ a n d ~}$ \\ CHENG Jie ${ }^{1, f}$ \\ ${ }^{1}$ Hubei Electric Power Survey \& Design Institute, Wuhan, Hubei Province, China \\ ${ }^{2}$ State Key Laboratory of Advanced Electromagnetic Engineering and Technology, Huazhong \\ University of Science and Technology, Wuhan, Hubei Province, China \\ a9071876@qq.com, bfz984843716@163.com, ywwu@mail.hust.edu.cn
}

Keywords: DC power flow; line loss; improved DC power flow; stochastic simulation

Abstract. Two methods, including standard DC power flow model and the improved DC power flow model with iteration process are introduced in this paper for line loss calculation. A line loss calculation model is established based on these two DC power flow models. In order to investigate the accuracy of the DC models, 6 systems are tested with stochastic simulation procedure. Results show that the improved DC power flow model with iteration process preserves the linearity of standard DC power flow and it also gains faster convergence speed than AC power flow model.

\section{Introduction}

Line loss is an important technological and economic indicators of power enterprises, and it reflects planning, production and operation management level of power network [1]. Theoretical line loss is generally obtained by AC power flow, but it is complicated and time-consuming due to nonlinear equations and mass data.

DC power flow model possesses linear, non-complex properties which appeal to analytical and computational usage in power system. Solutions of DC model are non-iterative, simple and efficient. DC model is widely used in static security analysis [2,3], electricity market and economic dispatch $[4,5,6]$. And DC power flow model achieves high accuracy under certain conditions [7], while it is rarely used in line loss calculation $[8,9]$.

DC power flow models are inherently approximate, and their accuracies are very system and case dependent [10]. Aiming at improving the accuracy, a number of methods derived from standard DC power flow model were presented. Reference [11] presents a method similar to DC power flow based on a known interface flow to correct node injections and admittance matrix. Reference [12] considers the active line loss, which is ignored in standard DC power flow model, with equivalent loads at both ends of the branch. Modified methods mentioned above gain good effect in specific conditions, while few accuracy tests about line loss calculation have been reported.

Given the above, this paper fulfils two main tasks. Firstly, it proposes a line loss calculation model based on two DC power flow models, including standard DC power flow model and the improved DC power flow model algorithm considering network loss. Secondly, it investigates the accuracy of the line loss calculation model.

\section{Standard DC Power Flow Model and Line Loss Calculation Model}

\section{Standard DC Power Flow Model}

Ignoring parallel branches of branch $i-j$ and then AC power flow equations are

$$
\left\{\begin{array}{l}
P_{i j}=\left(U_{i}^{2}-U_{i} U_{j} \cos \theta_{i j}\right) g_{i j}-U_{i} U_{j} b_{i j} \sin \theta_{i j} \\
Q_{i j}=-U_{i} U_{j} g_{i j} \sin \theta_{i j}-\left(U_{i}^{2}-U_{i} U_{j} \cos \theta_{i j}\right) b_{i j}
\end{array}\right.
$$


Where $P_{i j}$ and $Q_{i j}$ are active power and reactive power flowing from node $i$ to node $j ; U_{i}$ and $U_{j}$ are voltages of node $i$ and $j ; \theta_{i j}$ is the phase angle difference between node $i$ and $j$, that is $\theta_{i j}=\theta_{i}-\theta_{j} ; g_{i j}$ and $b_{i j}$ are conductance and susceptance of branch $i-j$.

According to the characteristics that node voltage is close to the rated voltage, phase angle difference is very small between two nodes of a branch and resistance is much smaller than reactance in the same branch when power network works normally, therefore standard DC power flow assumes that $U_{i}=U_{j}=1, \quad \sin \theta_{i j}=\theta_{i j}, \quad \cos \theta_{i j}=1, \quad g_{i j}=0$, Eq. 1 can be simplified as

$$
\left\{\begin{array}{l}
P_{i j}=-b_{i j} \theta_{i j}=\frac{\theta_{i j}}{x_{i j}} \\
Q_{i j}=0
\end{array}\right.
$$

Where $x_{i j}$ is the reactance of branch $i-j$.

For power network with $n+1$ nodes, node $N$ is set to be slack bus, and $N=n+1$. Then standard DC power flow equations are

$$
\boldsymbol{P}=\boldsymbol{B}_{0} \boldsymbol{\theta}
$$

Where $\boldsymbol{P}$ and $\boldsymbol{\theta}$ are n-dimensional active power vector and n-dimensional node voltage phase angle matrix of all buses except the slack bus; $\boldsymbol{B}_{0}$ is an $n$-dimensional admittance matrix which considers branch reactance only.

Elements in $\boldsymbol{B}_{0}$ are

$$
\left\{\begin{array}{l}
\boldsymbol{B}_{0}(i, i)=\sum_{j \in i, j \neq i} \frac{1}{x_{i j}} \\
\boldsymbol{B}_{0}(i, j)=-\frac{1}{x_{i j}}
\end{array}\right.
$$

\section{Line Loss Calculation Model Based on DC Power Flow}

Branches are regarded as lossless branches in standard DC power flow model with no reactive power. In order to calculate line loss, [13] brings in scale factor $\alpha_{i j}$ to take the ignored reactive power into account. Then, line loss calculation model based on DC power flow is as

$$
\begin{aligned}
& P_{\mathrm{loss}, i j}=S_{i j}^{2} r_{i j}=\left(\alpha_{i j} P_{i j}\right)^{2} r_{i j} \\
& \alpha_{i j}=\frac{S_{i j}}{P_{i j}}=\sqrt{1+\frac{r_{i j}^{2}}{x_{i j}^{2}}}
\end{aligned}
$$

Where $S_{i j}$ is the apparent power of branch $i-j, r_{i j}$ is the resistance of branch $i-j$, and $\alpha_{i j}$ is the scale factor between apparent power amplitude and active power of branch $i-j$.

Total line loss of the whole network is

$$
P_{\mathrm{loss}, \Sigma}=\sum_{i-j \in \Omega} P_{\mathrm{loss}, i j}
$$

Where $P_{\text {loss }, \Sigma}$ is theoretical line loss of the whole network, and $i-j \in \Omega$ represents all branches of the network. 


\section{The Improved DC Power Flow Model with Iteration Process}

\section{Network Loss Equivalent Load Model}

Reference [12] introduces network loss equivalent load model into DC power flow model in order to improve accuracy of standard DC power flow. Based on the same network loss equivalent load model, reference [13] proposes a DC optimal power flow algorithm. Then this paper intends to introduce network loss equivalent load model into line loss calculation with DC power flow model.

Fig. 1 shows the network loss equivalent load model, the model takes active power loss $P_{\text {loss }, i j}$ of branch $i-j$ into account and that is ignored in standard DC power flow model. At the same time, $P_{\text {loss }, i j}$ is divided equally into two parts in both ends of branch $i-j$.

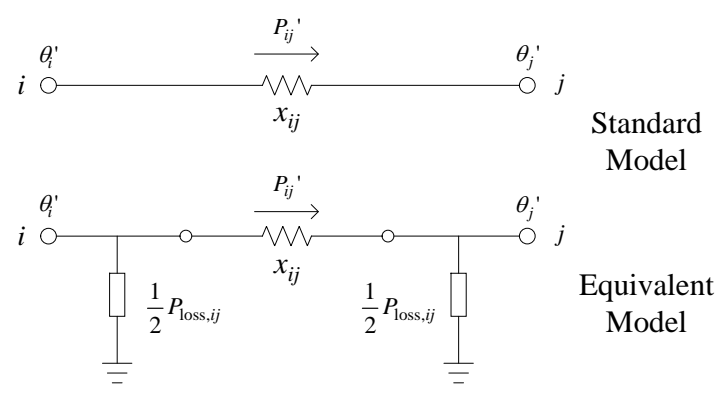

Fig. 1 Equivalent load model of network loss

In the network loss equivalent load model, formula of branch power flow is

$$
P_{i j}{ }^{\prime}=\frac{\theta_{i}{ }^{\prime}-\theta_{j}{ }^{\prime}}{x_{i j}}=\frac{\theta_{i j}{ }^{\prime}}{x_{i j}}
$$

It shares the same form with standard DC power flow model described above.

\section{The Improved DC Power Flow Model}

The improved DC power flow model with iteration process is based on network loss equivalent load model. Difference between the improved DC power flow model and standard DC power flow model is that the former one takes active power loss $P_{\text {loss }, i j}$ into account, and $P_{\text {loss }, i j}$ is used to modify node injection power. The improve DC model proceeds as following steps.

Step1. Preparing basic data for standard DC power flow, including node injection power vector $\boldsymbol{P}^{\boldsymbol{\prime}^{(1)}}=\left[P_{1}{ }^{\prime}, P_{2}{ }^{\prime}, \cdots, P_{n}{ }^{\prime}\right]^{\mathrm{T}}$, node admittance matrix $\boldsymbol{B}_{0}$ and voltage phase angle vector $\boldsymbol{\theta}^{\prime(1)}=\left[\theta_{1}{ }^{\prime}, \theta_{2}{ }^{\prime}, \cdots, \theta_{n}{ }^{\prime}\right]^{\mathrm{T}}$, set iteration times $k=1$, set iteration precision $\varepsilon=\varepsilon_{0}$, and set $P_{\mathrm{los}, i j}^{(0)}=0$ for all branches;

Step2. Solve equations $\boldsymbol{P}^{(k)}=\boldsymbol{B}_{0} \boldsymbol{\theta}^{(k)}$ and get branch power flow of all branches, combine these with Eq. 5, then line loss $P_{\text {loss } i j}^{(k)}$ can be calculated;

Step3. Judge convergence of current iteration, if all branches satisfy

$$
\left|P_{\text {loss }, i j}^{(k)}-P_{\text {loss }, i j}^{(k-1)}\right|<\varepsilon \cdot P_{\text {loss }, i j}^{(k-1)}
$$

Then this iteration converges, iterations are completed, otherwise, move to Step4;

Step4. Calculate equivalent load $P_{\text {equ }, i}^{(k)}$ and its vector $\boldsymbol{P}_{\text {equ }}^{(k)}$ :

$$
\begin{aligned}
& P_{\text {equ }, i}^{(k)}=\sum_{j \in i, j \neq i} \frac{P_{\text {loss }, i j}^{(k)}}{2} \\
& \boldsymbol{P}_{\text {equ }}^{(k)}=\left[P_{\text {equ }, 1}^{(k)}, P_{\text {equ }, 2}^{(k)}, \cdots, P_{\text {equ }, n}^{(k)}\right]^{\mathrm{T}}
\end{aligned}
$$

Step5. Calculate the new node injection power vector 


$$
\boldsymbol{P}^{\mathbf{\prime}^{(k+1)}}=\boldsymbol{P}^{\mathbf{\prime}^{(1)}}-\boldsymbol{P}_{\text {equ }}^{\mathbf{\prime}^{(\mathrm{k})}}
$$

Step6. Update literation times $k=k+1$, and if $k>k_{\max }$, literation times reaches the upper limit, and it means the equations are not convergent this time and it comes to the end the run, otherwise, return to Step2.

\section{Line Loss Calculation}

When the calculation process comes to the end, calculate loss of the network with Eq. 13 if the equations are convergent

$$
P_{\text {loss }, \Sigma}=\sum_{i-j \in \Omega} P_{\text {loss }, i j}^{(k)}
$$

While if the equations are not convergent, loss of the network can be calculated with branch line loss in the first iteration, that is

$$
P_{\mathrm{loss}, \Sigma}=\sum_{i-j \in \Omega} P_{\mathrm{loss}, i j}^{(1)}
$$

Results calculated by Eq. 14 equals that of Eq. 5 to Eq. 7 for the same network.

\section{Assessing DC Models Accuracy}

\section{Case Simulation Design}

In order to analysis the accuracy of the standard DC power flow model and the improved DC power flow model in the fields of line loss calculation, this paper designs a test procedure as Fig. 2. Six systems are tested in the paper, including WSCC-9, IEEE-30, European-89, IEEE-118, Polish-2383 and Polish-3120.

Take IEEE-30 system as an example to illustrate the test procedure in Fig. 2. Firstly, case data of IEEE-30 system are to be prepared. Secondly, set the change interval of node injection power as [-50\%,50\%], which means each node injection power of all 30 nodes are changing with variation from $-50 \%$ to $50 \%$. Thirdly, secondary power balance procedure should be carried out because the random variation in the second step may produce unbalanced power, and method mentioned in [14] is taken in this paper for the secondary power balance procedure. Fourthly, calculate the AC power flow, standard DC power flow and the improved DC power flow and their corresponding losses. Fifthly, analysis the relative error between AC model and DC models. Finally, repeat above steps M times.

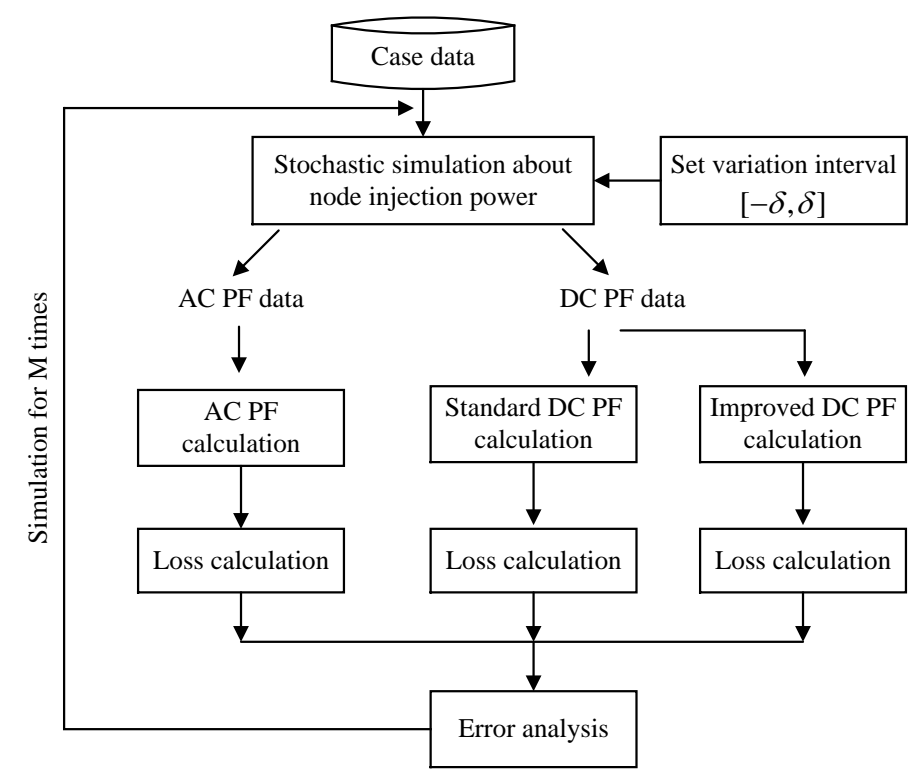

Fig. 2 Accuracy test procedure about two DC models 


\section{Error Analysis}

For the same test system, in the $i^{\text {th }}$ simulation, results of loss calculation are expressed as $P_{\text {lossDCl, } i}$ for standard DC power flow, $P_{\text {lossDC } 2, i}$ for the improved DC power flow and $P_{\text {lossAC }, i}$ for AC power flow. Then relative errors in the $i^{\text {th }}$ simulation between DC models and AC model can be calculated with

$$
\begin{gathered}
e_{r 1, i}=\frac{\left|P_{\text {lossDC1 }, i}-P_{\text {lossAC }, i}\right|}{P_{\text {lossAC }, i}} \\
e_{r 2, i}=\frac{\left|P_{\text {lossDC } 2, i}-P_{\text {lossAC }, i}\right|}{P_{\text {lossAC }, i}}
\end{gathered}
$$

When simulation procedure is repeated for $\mathrm{M}$ times, average relative errors between DC models and AC model are

$$
\begin{aligned}
& \bar{e}_{r 1}=\frac{1}{\mathrm{M}} \sum_{i=1}^{\mathrm{M}} \frac{\left|P_{\mathrm{lossDC}, i}-P_{\mathrm{lossAC}, i}\right|}{P_{\mathrm{lossAC}, i}} \\
& \bar{e}_{r 2}=\frac{1}{\mathrm{M}} \sum_{i=1}^{\mathrm{M}} \frac{\left|P_{\mathrm{lossDC}, i}-P_{\mathrm{lossAC}, i}\right|}{P_{\mathrm{lossAC}, i}}
\end{aligned}
$$

Set number of simulation times $\mathrm{M}=500$, set the change interval as [-50\%,50\%] and set iteration precision $\varepsilon=0.0001$, then relative errors between DC models and AC model are shown in Table 1 .

Table 1 Average relative error study

\begin{tabular}{|c|c|c|}
\hline System & $\bar{e}_{r 1}$ & $\bar{e}_{r 2}$ \\
\hline WSCC-9 & $4.9 \%$ & $5.4 \%$ \\
\hline IEEE-30 & $10.9 \%$ & $8.6 \%$ \\
\hline European-89 & $2.5 \%$ & $2.3 \%$ \\
\hline IEEE-118 & $4.0 \%$ & $1.0 \%$ \\
\hline Polish-2383 & $8.2 \%$ & $1.1 \%$ \\
\hline Polish-3120 & $3.1 \%$ & $6.5 \%$ \\
\hline
\end{tabular}

Average relative errors between DC models and AC model are very small in general. Max average relative error between standard DC power flow and AC power flow is 10.9\% in IEEE-30 system, and that of the improved DC power flow is $8.6 \%$ in IEEE-30 system. For the tested 6 systems, 2 out of the 6 systems have gotten higher accuracy with standard DC power flow, including WSCC-9 and Polish-3120; the other 4 systems archive higher accuracy with the improved DC power flow, including IEEE-30, European-89,IEEE-118 and Polish- 2383.

\section{Sensitivity Analysis}

Fig. 3 gives the sensitivity analysis results about standard DC model. When the change interval ranges from $[-10 \%, 10 \%]$ to $[-50 \%, 50 \%]$, average relative errors between standard DC power flow and $\mathrm{AC}$ power flow have not changed much for each of the 6 systems. Fig. 4 gives the sensitivity analysis results about the improved DC model. When the change interval ranges from [-10\%,10\%] to $[-50 \%, 50 \%]$, average relative errors between the improved DC power flow and AC power flow have not changed much for each of the 6 systems.

Sensitivity analysis results show that the two DC models are stable and reliable when calculating line loss. Furthermore, it's easy to find out that the improved DC power flow model gain higher accuracy in majority systems. 


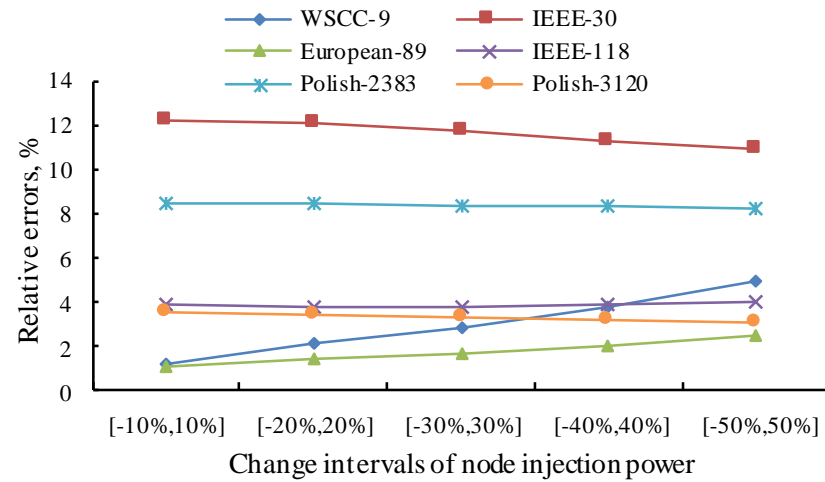

Fig. 3 Sensitivity analysis about standard DC model

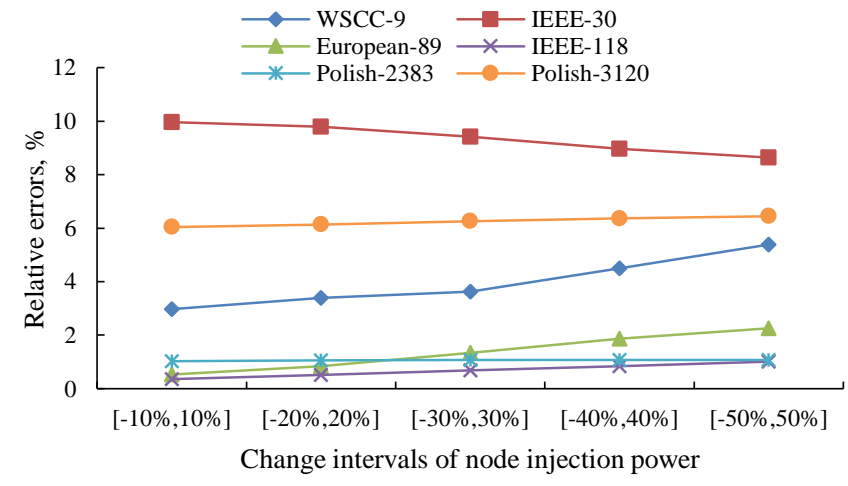

Fig. 4 Sensitivity analysis about the improved DC model

\section{Comprehensive Comparison of DC Models}

Both Standard DC power flow model and the improved DC power flow model achieve high accuracy in line loss calculation. In most cases, average relative errors of these two methods are less than $10 \%$. Majority of the cases show that the improved DC model gains higher accuracy.

Sensitivity analysis proves that these two DC models are stable and reliable when they are used for line loss calculation. Relative errors of both methods have not any trend of increasing when the simulation interval rises.

In the aspect of computing speed, it is well known that standard DC power flow needs to solve linear equation once only, and its solving procedure is simpler and more efficient than the AC model. As for the improved DC power flow model, it takes branch line loss into account, and the solving procedure of the improved model requires several iterations to get its branch power flow, but it repeats to solve linear equations in every iteration, that is to say, the solving procedure of the improved DC model retains the simple and efficient characteristics of standard model. Furthermore, a number of cases show that the improved DC model can converge within 2-5 times iterations when the iteration precision is set as $\varepsilon=0.0001$, which is less than that of AC power flow usually.

\section{Conclusion}

Line loss calculation based on DC power flow models is researched in this paper. After analyzing the theory and characteristics of two DC models, including standard DC power flow model and the improved DC power flow model with iteration process, a line loss calculation model based on DC models is introduced in this paper.

A test procedure is designed here to investigate the accuracy of these two DC models. Five intervals ranging from $[-10 \%, 10 \%]$ to $[-50 \%, 50 \%]$ of node injection power are tested with each of the six systems. Results show that standard DC model and the improved DC model are both stable in line loss calculation, while the improved DC model achieves higher accuracy than standard DC model in most cases.

The improved DC power flow model with iteration process preserves the linearity of standard DC power flow and it also gains faster convergence speed than AC power flow model. It can be applied for line loss calculation instead of AC model in the situation that calls for simple computing process and faster calculation speed with acceptable accuracy.

\section{References}

[1] K.K. Zhang, X.Y. Yang, C.R. Bu, R. Wei, C.J. Liu, Y. Yang and Y. Chen: Proceedings of the CSEE Vol. 33(2013), p. 92-97

[2] F. Li, H.C. Li, Y.J. Yu, and S.M. Feng: Automation of Electric Power Systems Vol. 37(2013), p. 75-80

[3] Y. Shen, Q. Xia and C.Q. Kang: Automation of Electric Power Systems Vol. 27(2003), p. 13-17 
[4] B.F. Hobbs, C.B. Metzler and J.S. Pang: IEEE Trans. on Power Syst. Vol. 15(2000) p. 638-645

[5] M. Chávez-Lugo, C.R. Fuerte-Esquivel, C.A. Cañizares and V.J. Gutierrez-Martinez: IEEE Trans. on Power Syst. Vol. 31(2016), p. 3358-3368

[6] Q. Xia, B.M. Zhang, C.Q. Kang, N.D. Xiang and Y.H. Song: Power System Technology Vol. 21(1997), p. 61-65

[7] J.Q. Zhao, J.L. Ye and Y Deng: Power System Technology Vol. 36(2012), p. 147-152

[8] J.H. Huang, J. Tan, J.Y. Yang, H. Li and J. Han: Automation of Electric Power Systems Vol. 38(2014), p. 39-42

[9] J.K. Wu, Y.H. Li, H.L. Zhang and G.T. Chen: Modern Electric Power Vol. 25(2008), p. 8-12

[10] B. Stott, J. Jardim and O. Alsac: IEEE Trans. on Power Syst. Vol. 24(2009), p. 1290-1300

[11]H.C. Li, Y.J. Yu, Z.H. Gao, F. Li and S.M. Feng: Automation of Electric Power Systems Vol. 37(2013), p. 128-133

[12]H.F. Wang, Y. Wang, C. Gao, L. Xu, J.X. Hou and X.H. Tao: Automation of Electric Power Systems Vol. 39(2015), p. 99-103

[13] T.Y. He, Z.N. Wei, G.Q. Sun, Y.H. Sun, H.X. Zang and Q. Gao: Automation of Electric Power Systems Vol. 40(2016), p. 58-64

[14] Y. Wang, J.X. Hou, S.Y. Ma, C. Zheng, X.F. Xu and Z.G. Yang: Power System Technology Vol. 34(2010), p. 100-104 